\title{
Is Ropivacaine a Non-cardiotoxic Local Anesthetic? Study of the Frequency- dependent Cardiotoxicity of Ropivacaine in an Experimental Porcine Model
}

\author{
García S1, Varela O', Melone A', Callejo D1, Quintela $\mathbf{O}^{2}$, Zaballos $\mathbf{M}^{1}{ }^{2}$, \\ 1- (Department of Anaesthesia, Hospital Gregorio Marañón (Madrid, Spain) \\ 2- Faculty of Medicine, Universidad Complutense de Madrid (Spain)
}

\section{BACKGROUND AND GOAL OF STUDY}

Ropivacaine $(R)$ is considered a less cardiotoxic local anesthetic (LA) in comparison to others. However, clinical reports have been described, in which its accidental administration has caused severe arrhythmias, ventricular tachycardia and even cardiac arrest. The cardiotoxicity of $R$ is related to the inhibition of the fast inward current during the depolarization of cardiac cells. Studies in vitro have shown that $R$ toxicity increases in a use-dependent tendency. Our aim was to characterize an in vivo model to evaluate cardiotoxicity of $R$.

\section{MATERIAL AND METHODS}

Eight anesthetized and instrumentalized pigs were studied. Three quadripolar catheters were positioned into the high right atrium, the right ventricular apex, and to the His bundle recording area. After a period of stabilization pacing was performed at a current strength of $30 \mathrm{~mA}$ with a programmable stimulator. Right ventricular pacing was performed for at least 10 beats at a cycle lengths of 400 and $500 \mathrm{~ms}$. This pacing protocol was performed immediately before and at $1,5,10,15$ and 30 minutes of the administration of $R(6 \mathrm{mg} / \mathrm{kg}$ in two animals and $5 \mathrm{mg}$ in 6 animals). Statistics: Analysis of variance for repeated measures

\section{RESULTS}

Two animals, which received $6 \mathrm{mg} / \mathrm{kg}$ of $\mathrm{R}$, died due to severe hypotension. The rest of the animals received 5 $\mathrm{mg} / \mathrm{kg}$. Plasma $\mathrm{R}$ levels ranged between $7,940-5,450$ $\mathrm{ng} / \mathrm{dl}$ from 5 to $30 \mathrm{~min}$ respectively. Ropivacaine induced an intense toxicity effect in sinusal rhythm as well as in stimulated rhythm. After $\mathrm{R}$ administration there was an important prolongation in QRS interval in sinusal rhythm: from $67 \pm 9$ to $97 \pm 18 \mathrm{~ms} ; \mathrm{p}=0,0001$; ( $\triangle 45 \%$ ) and at paced cycle length of $400 \mathrm{~ms}$ : from $99 \pm 6 \mathrm{~ms}$ to $355 \pm 73 \mathrm{~ms} ; \mathrm{p}=0,0001) ;(\Delta 258 \%$ ) (Figure). Ten minutes after $R$ administration, still persisted an intense increment up to $90 \%$ in stimulated QRS, however, QRS interval in sinusal rhythm showed values in normal range ( $82 \pm 6 \mathrm{~ms})$.

\section{CONCLUSIONS}

$\mathrm{R}$ has been associated with a huge cardiotoxic effect that has been shown with fast frequencies of stimulation. This experiment has unmasked a hidden cardiotoxic phenomenon that persists intensely even after 10 minutes since the administration of the drug. These findings suggest that after an accidental $R$ dose precaution measures should be maximized as well as avoiding sympathetic simulation until cardiotoxicity parameters are completely restored.

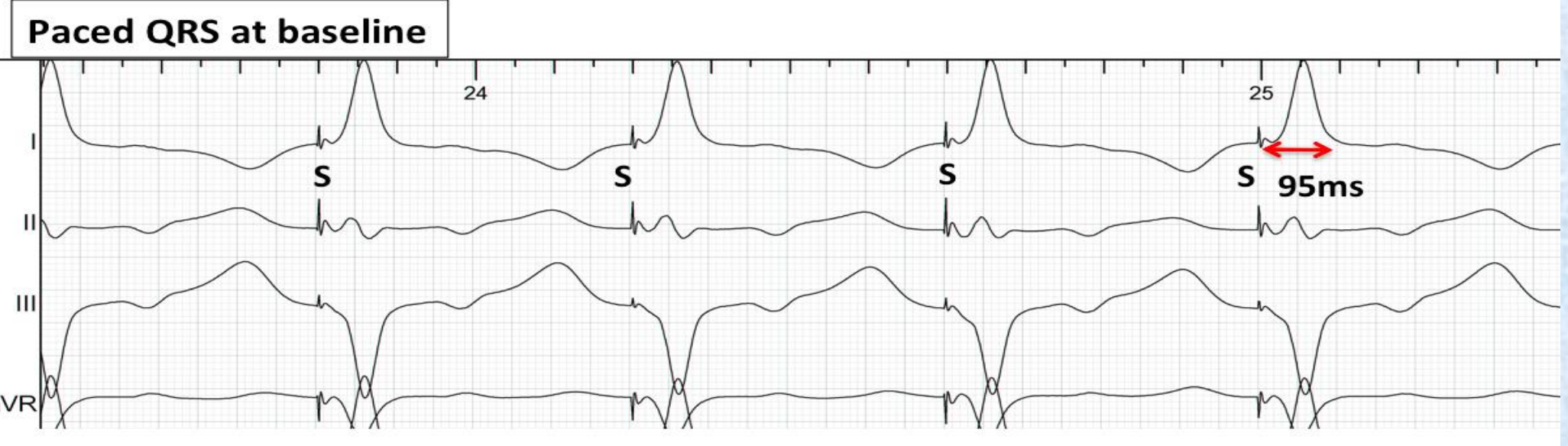

Paced QRS 5 min after ropivacaine administration

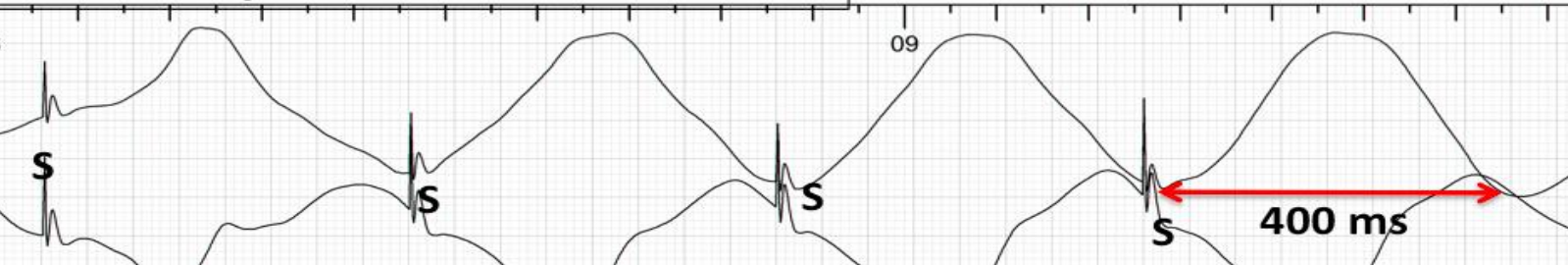

Figure 1: An example of the frequency-dependent protocol after ropivacaine administration in the same animal. At baseline de stimulated QRS duration was $95 \mathrm{~ms}$; at the bottom after ropivacaine the de stimulated QRS duration was increased to $400 \mathrm{~ms}$.

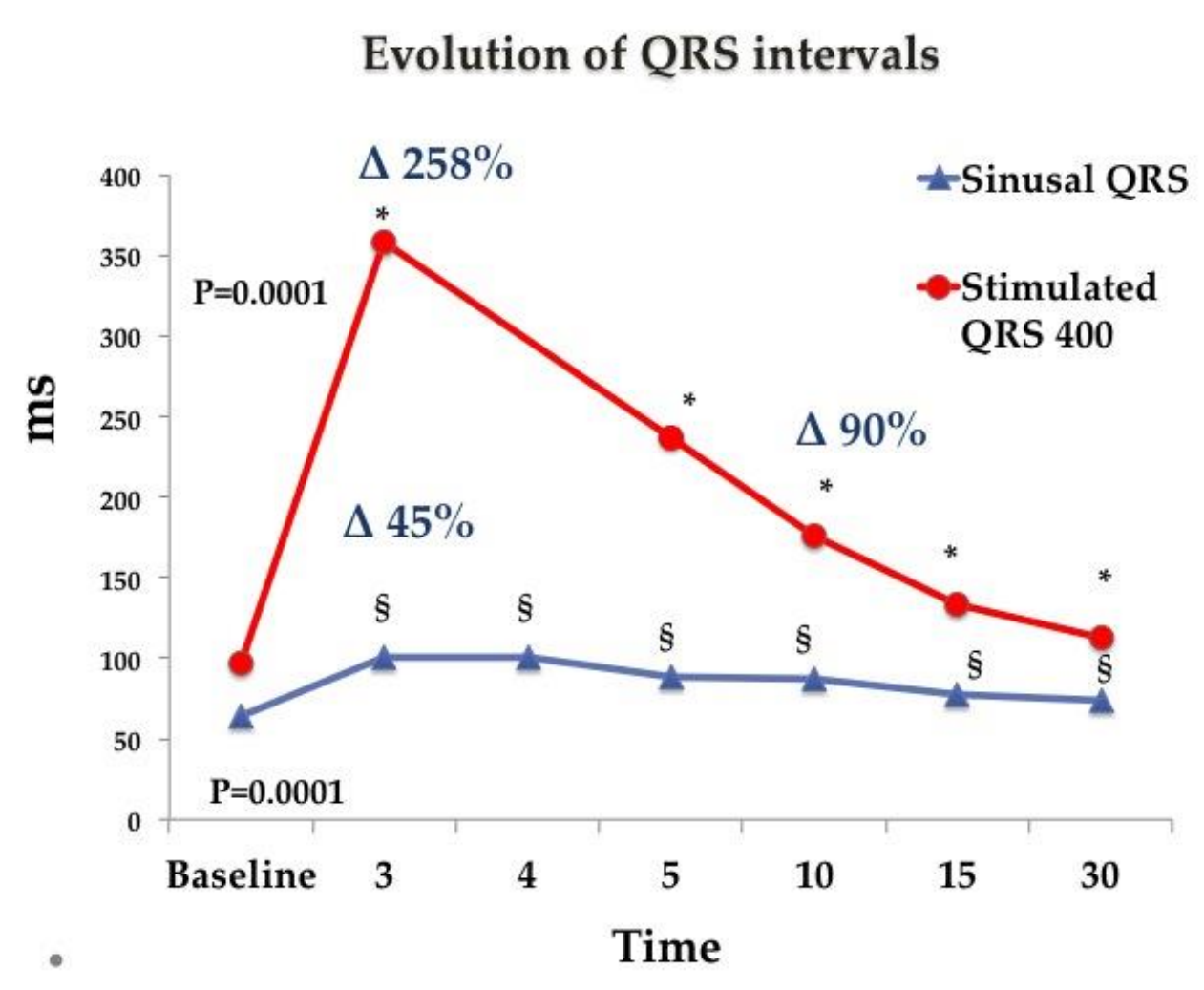

Figure 2. After ropivacaine administration a mean increase of $258 \%$ was observed in stimulated QRS and $45 \%$ in sinusal QRS. At ten minutes we still have an intense effect on ventricular conduction and the increase in stimulated QRS is still $90 \%$. 